\title{
Use of Botulinum Toxin in Treating Rosacea: A Systematic Review
}

\author{
Hanlin Zhang (1)* \\ Keyun Tang $\mathbb{D}^{*}$ \\ Yuanzhuo Wang (D) \\ Rouyu Fang (D) \\ Qiuning Sun (iD)
}

Department of Dermatology, Peking Union Medical College Hospital, Beijing, People's Republic of China

*These authors contributed equally to this work
Correspondence: Qiuning Sun

Tel +86-|0-69|56|| 4

Fax +86-10-69|56|14

Email doctorjennyl@163.com
Background: Rosacea is a chronic skin disorder characterized by erythema, flushing, telangiectasia, papules and pustules, phymatous changes, and ocular involvement. The aim of this study was to examine all published research articles in which botulinum toxin was used to treat rosacea and to evaluate the efficacy and safety of this treatment.

Methods: PubMed, Embase, Cochrane Library, and Web of Science database were used to identify articles eligible for systematic review on March 26, 2021. Prospective or retrospective studies which directly used botulinum toxin to treat rosacea were included and reviewed.

Results: Nine studies were included in this systematic review. Two were randomized controlled trials, graded as Level 2 for the quality of evidence. The total number of participants was 130, and the number of participants in each study ranged from 1 to 25 . The improvement was observed in all studies in signs and symptoms compared with baseline. Adverse events were transitory and self-limited.

Conclusion: Botulinum toxin could have overall satisfying efficacy and safety in the treatment of rosacea, though limited by small sample size, imperfect study design, and short follow-up visits.

Keywords: botulinum toxin, rosacea, systematic review

\section{Introduction}

Rosacea is a chronic skin disorder characterized by erythema, flushing, telangiectasia, papules and pustules, phymatous changes, and ocular involvement. ${ }^{1-5}$ In 2012, a standard classification system for rosacea was developed by the National Rosacea Society Expert Committee. Four distinct subtypes of rosacea were established: erythematotelangiectatic, papulopustular, phymatous, and ocular rosacea. ${ }^{6}$ In 2017 , a phenotype-based approach for diagnosis and classification of rosacea was recommended, in which persistent centrofacial erythema and phymatous changes were two diagnostic features. ${ }^{7,8}$ Facial rosacea may affect one's professional, social, and family life. Skin care, sun protection, laser and pulsed light, topical or oral medications are major interventions for rosacea patients. ${ }^{9-11}$ However, treatment for severe or refractory rosacea remains challenging for dermatologists. ${ }^{12,13}$

As an injectable neuromodulator, botulinum toxin, especially type $\mathrm{A}$, is useful to improve skin conditions and cosmetic defects. ${ }^{14-19}$ Several reviews mentioned the use of botulinum toxin in rosacea treatment. ${ }^{10,20,21}$ However, the study design, formulations, dilution and dosing, outcome measurement, and efficacy and safety were different in these studies. The aim of this article is to examine all published research articles 
in which botulinum toxin was used to treat rosacea, and to evaluate the efficacy and safety of this treatment.

\section{Methods}

\section{Search Strategy and Study Selection}

This study was conducted in accordance with the PRISMA guidelines, and the protocol registered online in the PROSPERO on March 10, 2021 (ID: 242088; still being assessed by the editorial team). ${ }^{22}$ PubMed, Embase, Cochrane Library, and Web of Science database were used to identify articles eligible for systematic review on March 26, 2021, using the following strategy: (botulinum* OR botox) AND (rosacea) for PubMed and Embase, (*botulinum* OR botox) AND (rosacea) for Cochrane Library and Web of Science. Two researchers ( $\mathrm{H}$. Zhang and K. Tang) conducted an exhaustive search and selection independently for qualified papers, and a senior reviewer (Q. Sun) participated in order to reach consensus in case of disagreement. The inclusion criterion was prospective or retrospective studies directly using botulinum toxin to treat rosacea, with limits to English language articles and human subjects. Articles focusing on other topics or many diseases, animal experiments, trial registration, protocols, literature reviews, conference articles, and patents were all excluded.

\section{Data Extraction and Quality Assessment}

The following data were extracted from selected articles: author and year of publication, study design, number of participants and subtype of rosacea, commercial forms, dilution and dosing, outcome measurement, findings, side effects, and follow-up. For randomized controlled trials (RCTs), the risk of bias was assessed using the Cochrane Collaboration's tool. ${ }^{23}$ Non-randomized prospective studies were evaluated using the methodological index for non-randomized studies (MINORS). ${ }^{24}$ The methodologic quality and risk of bias of case series and case reports were evaluated by JBI Critical Appraisal Checklist. ${ }^{25}$ We attributed 0 points for No, 1 point for Unclear, and 2 points for Yes for each item. Total scores were calculated and recorded as percent of total. The studies were determined to present low, moderate, or high risk of bias if the percent of total was $\geq 80 \%, 60 \%-80 \%$, or $\leq 60 \%$, respectively. ${ }^{26}$ Levels of evidence were graded according to Oxford Centre for Evidence-Based Medicine 2011 Levels of Evidence (https://www.cebm.net/wp-content/uploads/2014/ 06/CEBM-Levels-of-Evidence-2.1.pdf). Quality assessment was performed by two independent researchers ( $H$. Zhang and $\mathrm{K}$. Tang), and disagreements were resolved by discussion.
Meta-analysis could not be performed mainly because of the study design and outcome heterogeneity. This study did not involve intervention or data collection in animal experiments or clinical trials. Thus, approval from an ethical committee was not needed.

\section{Results}

The result of the literature search was shown in Figure 1. Nine studies were included in this systematic review (Table 1). ${ }^{13,27-34}$ These studies were published between 2012 and 2020. Two studies were RCTs. The total number of participants was 130, and the number of participants in each study ranged from 1 to 25 .

In the nine studies, four focused on erythematotelangiectatic rosacea, two focused on erythematotelangiectatic or papulopustular rosacea, and three did not mention the subtype of rosacea. Botulinum toxin A was used in eight studies, including 4 OnabotulinumtoxinA, 3 AbobotulinumtoxinA, 1 IncobotulinumtoxinA, and 1 PrabotulinumtoxinA. One case report did not mention the formulation of botulinum toxin. ${ }^{32}$ Dilution and dosing varied in different studies, and the detailed dilution and dosing were listed in Table 1.

There were different categories of outcome measurements, qualitative and quantitative evaluations from doctors or patients. Antera 3D camera was used to take standardized photographs before and after treatments, and measure erythema quantification. Rosacea Clinical Scorecard (RCS) assessment and Clinician Erythema Assessment (CEA) score were used to determine the severity of rosacea symptoms. Corneometer, mexameter, reviscometer, and sebumeter were used to provide biophysical measurements. State SelfEsteem Scale (SSES) questionnaire, Patients selfassessment (PSA) scores, and the dermatology life quality index (DLQI) were used to evaluate the feeling of patients.

Overall, the improvement was observed in the nine studies in signs and symptoms of rosacea compared with baseline. In Bloom et al study with 25 participants, ${ }^{28}$ one withdrew, and nine had inconsistent follow-up visits, the reason for which was not provided. In Park et al study with 20 Korean patients, ${ }^{31}$ three patients reported undesired paralysis of facial muscles and stopped participating the study, although their symptoms were resolved without any special treatment. In Kim et al study with 24 participants, ${ }^{33}$ one patient was excluded because of protocol violation, intake of muscle relaxant for ankle sprain. Other side effects, such as pain, bruising, erythema, purpura, edema, were mild and self-limited. The follow-up varied in different studies, varying from 8 weeks to 9 months. 


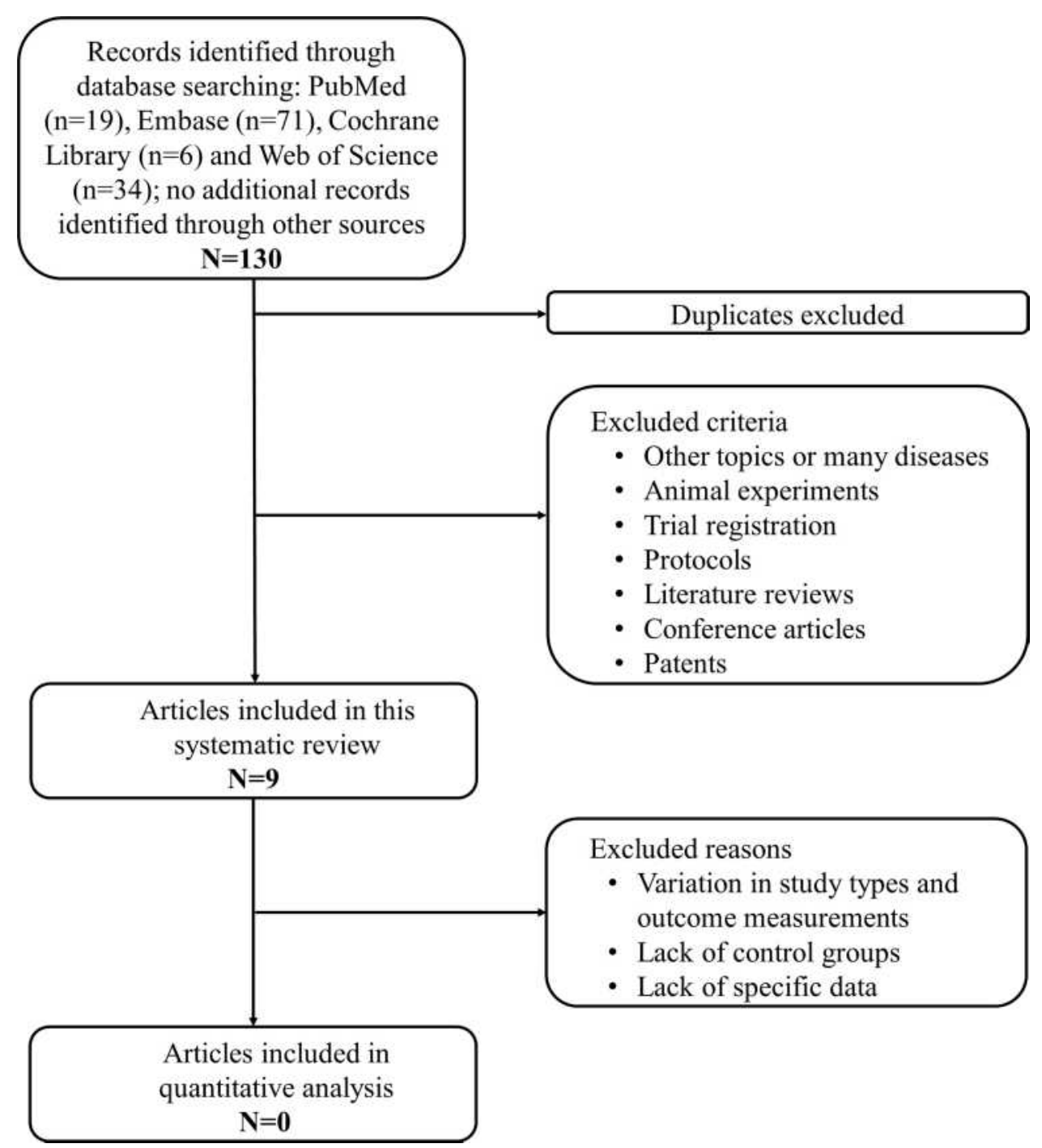

Figure I Study flow diagram.

As for the risk of bias, detailed evaluation results were shown in Supplementary Table 1-4. Three studies were categorized as low risk, four were moderate, and two were high. One RCT was at moderate risk, and the other was at high risk. Dayan et al study was judged as moderate because the blinding of outcome assessment was not clearly demonstrated. ${ }^{35} \mathrm{Kim}$ et al study was judged as high because the research funds and drugs were provided by Daewoong Pharmaceutical. ${ }^{33}$ As for the quality of evidence, two randomized controlled trials were Level 2, and other studies were Level 4, according to Oxford Centre for Evidence-Based Medicine 2011 Levels of Evidence.

\section{Discussion}

This systematic review of botulinum toxin in treating rosacea identified nine relevant articles, including two randomized controlled trials. There is variety among these studies regarding study design, number of participants, subtype of rosacea, commercial forms, dilution and dosing, and outcome measurement. Improvement in signs and symptoms of rosacea was observed in all studies compared with baseline. Side effects were all transitory and self-limited. Because of the variety of studies, it remains difficult to interpret these outcomes collectively.

Rosacea is a chronic cutaneous disorder primarily affecting the central face, with remissions and exacerbations. ${ }^{6}$ A variety of treatment modalities have been reviewed, including skin care and cosmetic treatments, topical therapies, oral therapies, laser- and light-based therapies, injection therapies, and combination therapies, based on their anti-demodex, anti-inflammatory, and anti-angiogenesis effects. ${ }^{36-39}$ However, recalcitrant, refractory, and persistent rosacea remains 


\begin{tabular}{|c|c|c|c|}
\hline 总 & $\frac{+}{\bar{g}}$ & $\frac{\bar{\sigma}}{\stackrel{\sigma}{\omega}}$ & 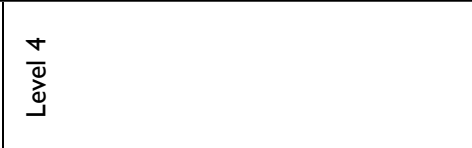 \\
\hline 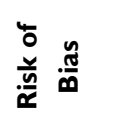 & $\stackrel{3}{9}$ & 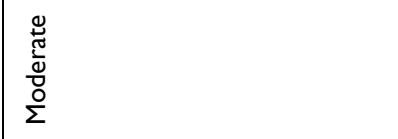 & 3 \\
\hline 产 & 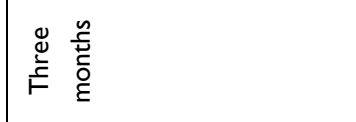 & 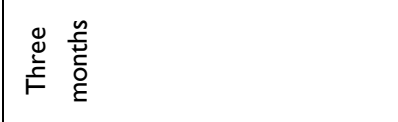 & 总 \\
\hline 怤离 & $\stackrel{0}{z}$ & 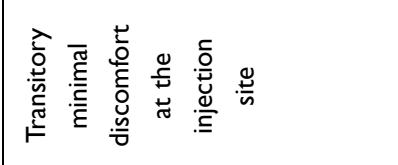 & 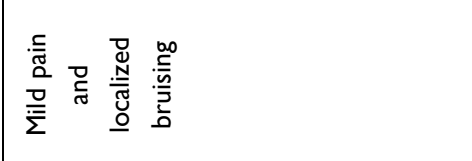 \\
\hline 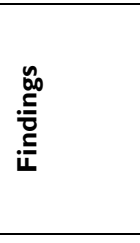 & 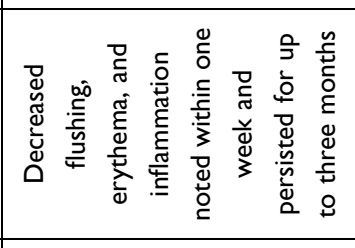 & 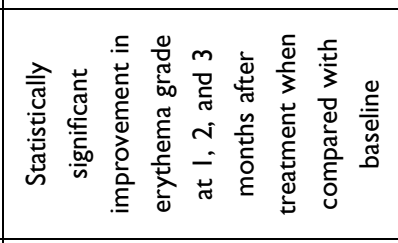 & 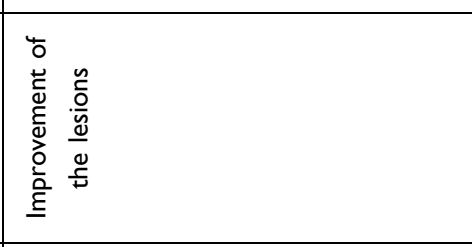 \\
\hline 䓂 & 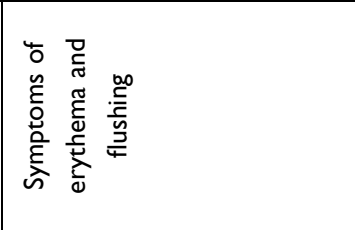 & 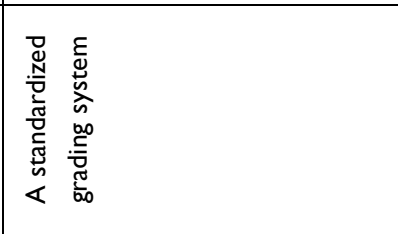 & 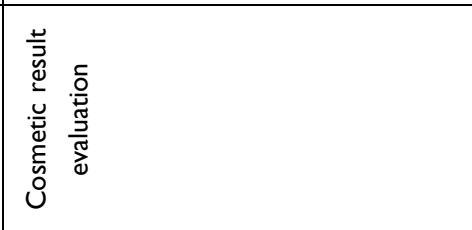 \\
\hline 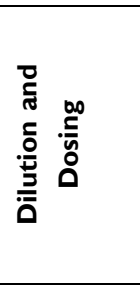 & 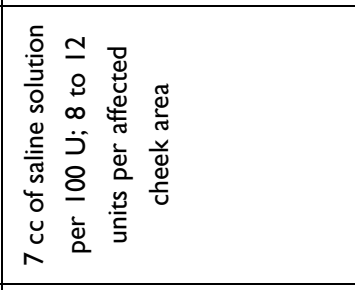 & 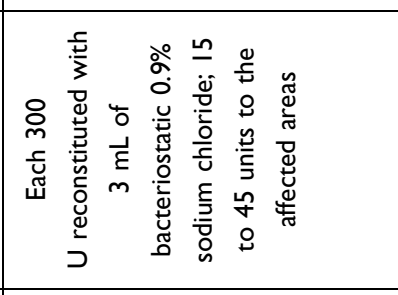 & 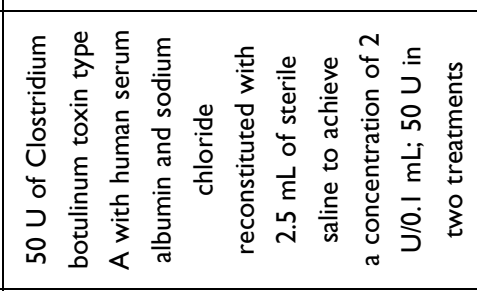 \\
\hline . & 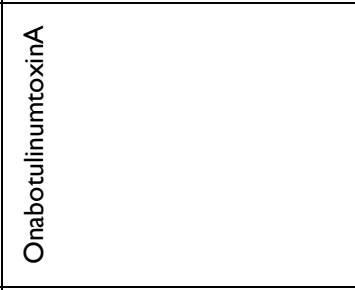 & 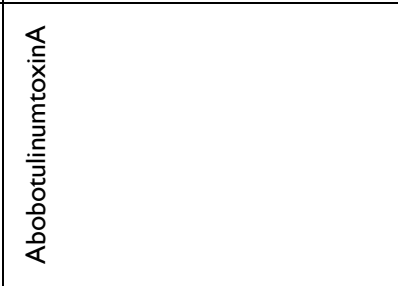 & 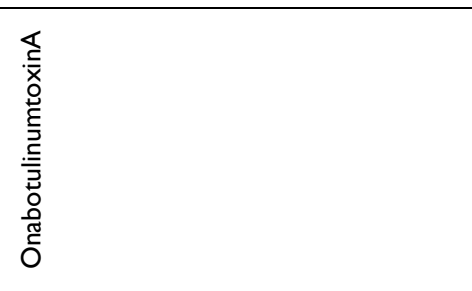 \\
\hline 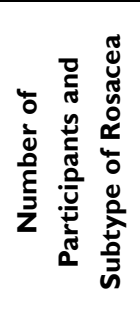 & 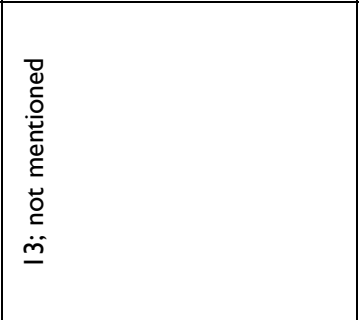 & 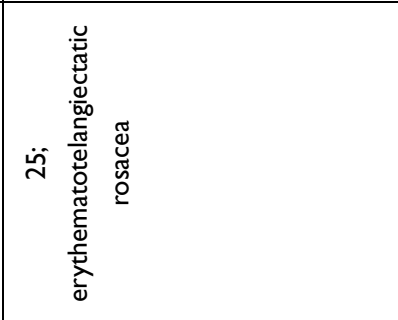 & 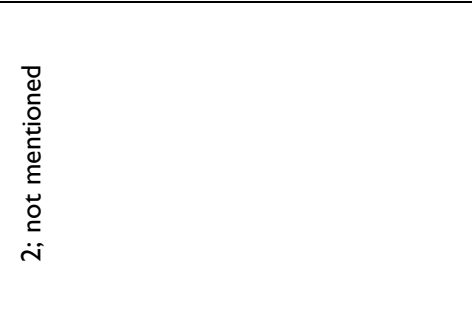 \\
\hline 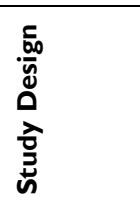 & 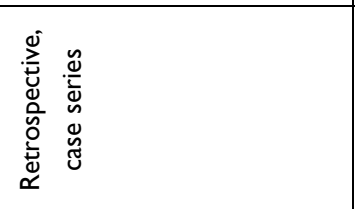 & 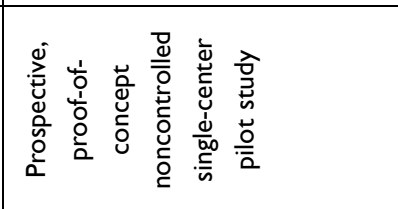 & 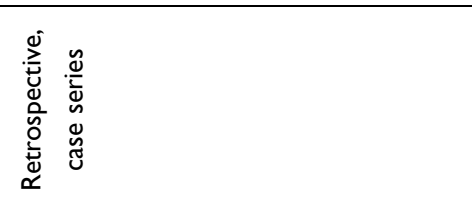 \\
\hline 蒿 & 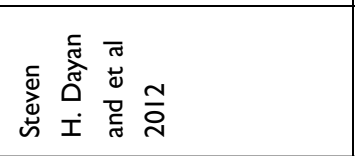 & 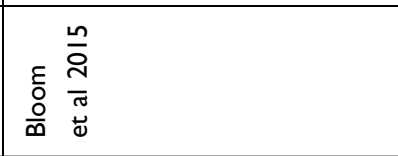 & 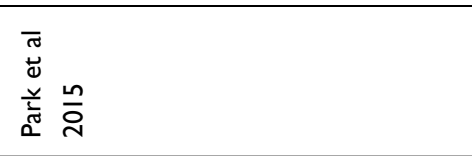 \\
\hline
\end{tabular}




\begin{tabular}{|c|c|}
\hline 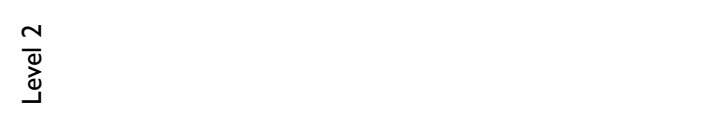 & 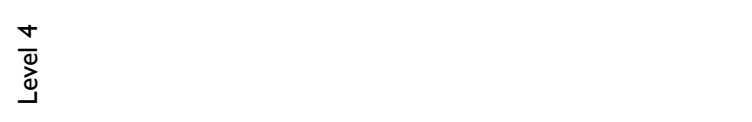 \\
\hline 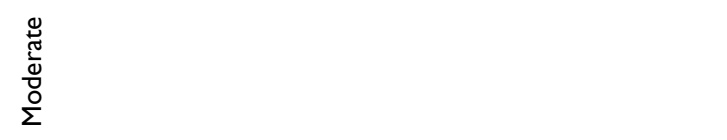 & 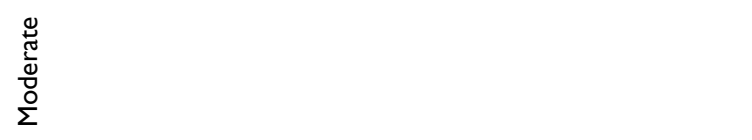 \\
\hline$\stackrel{\frac{\tilde{\sigma}}{0}}{\frac{\pi}{3}}$ & 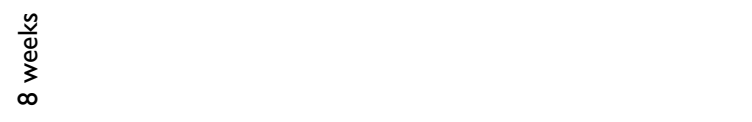 \\
\hline$\stackrel{\circ}{z}$ & 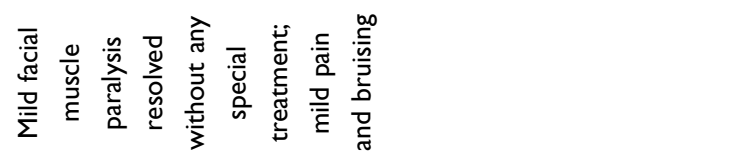 \\
\hline 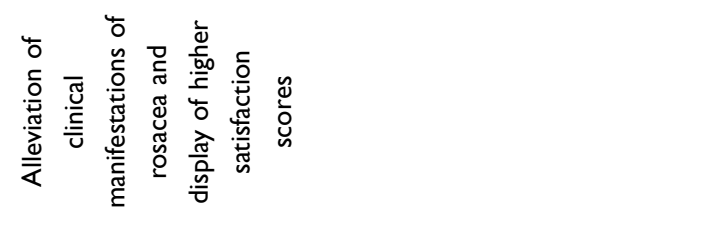 & 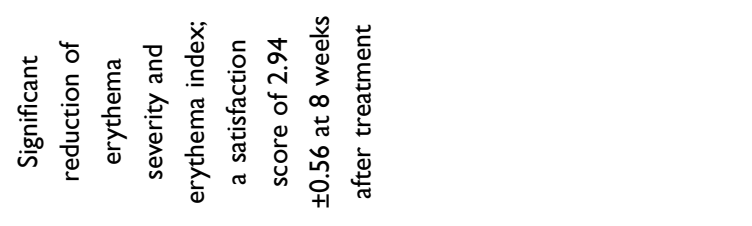 \\
\hline 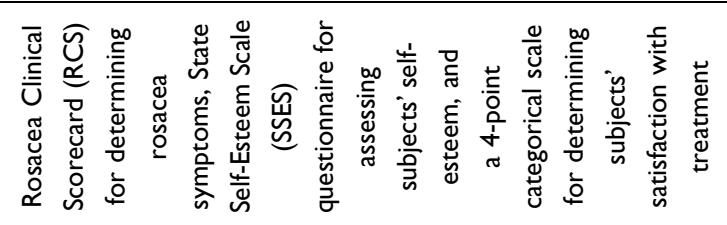 & 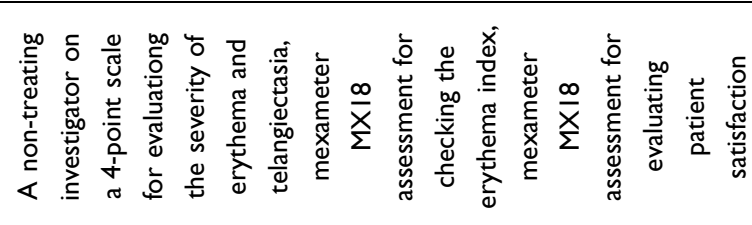 \\
\hline 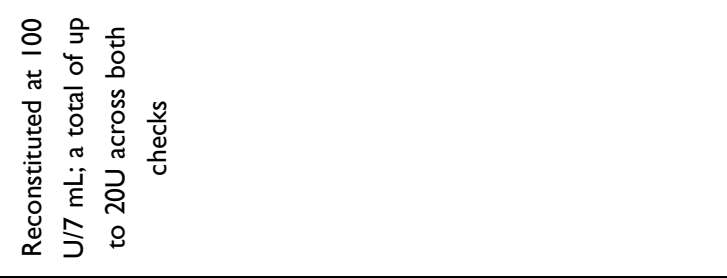 & 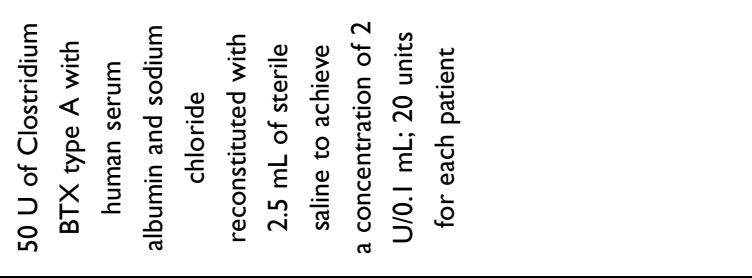 \\
\hline 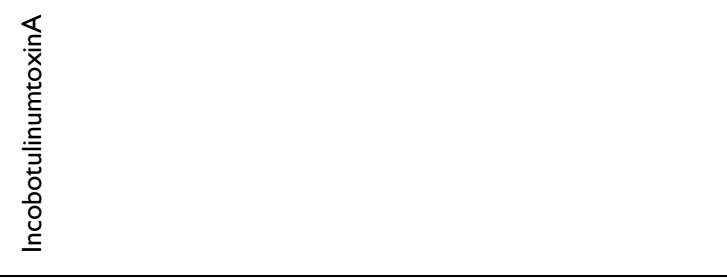 & 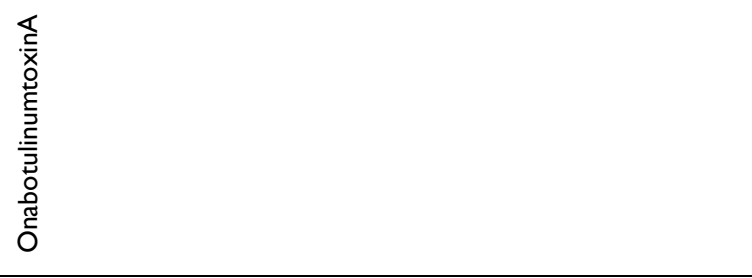 \\
\hline 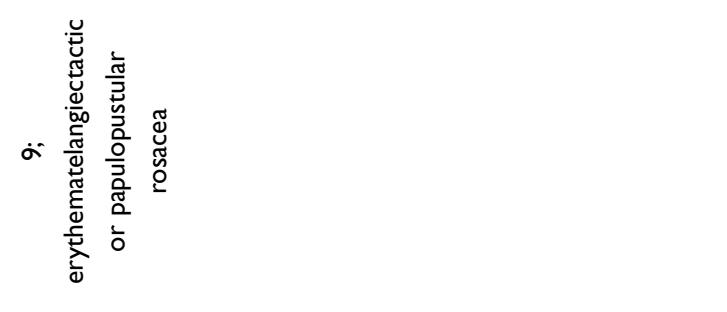 & 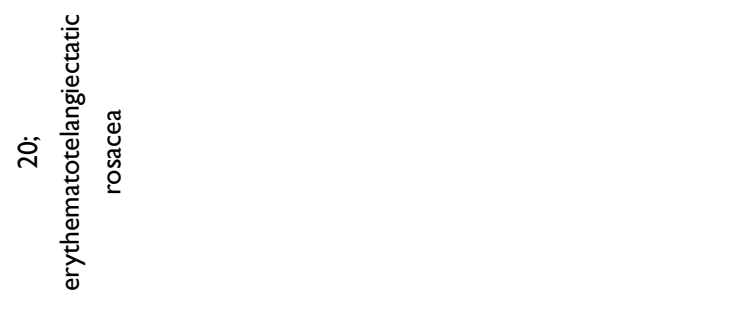 \\
\hline 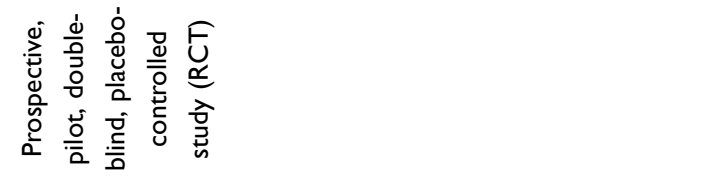 & 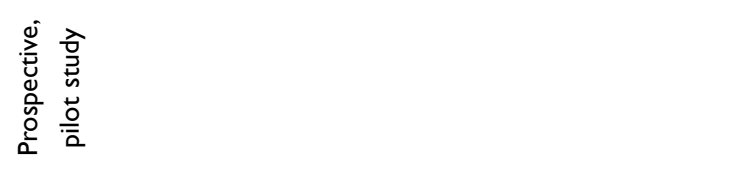 \\
\hline 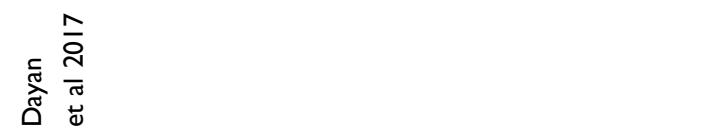 & 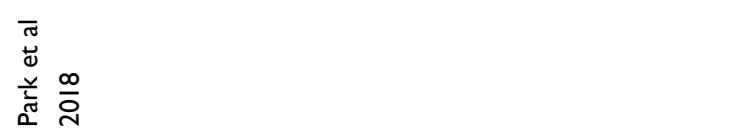 \\
\hline
\end{tabular}




\begin{tabular}{|c|c|c|}
\hline 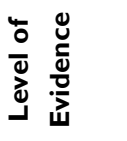 & 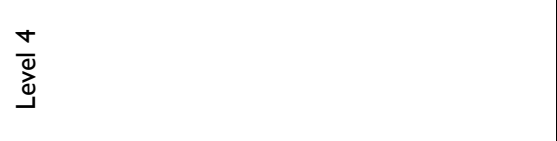 & $\stackrel{\sim}{\stackrel{d}{\nu}}$ \\
\hline 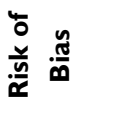 & 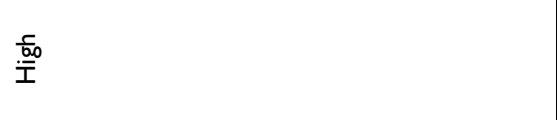 & 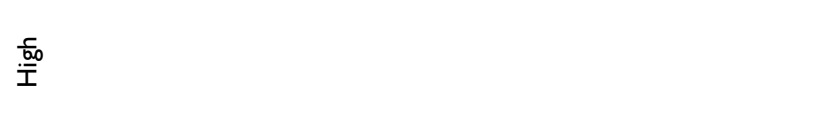 \\
\hline כ) & 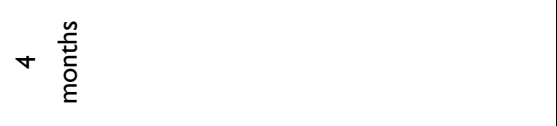 & $\simeq \frac{\stackrel{\tilde{\Perp}}{\varpi}}{3}$ \\
\hline 造 & 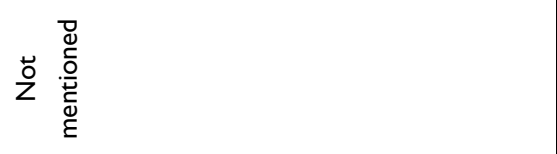 & 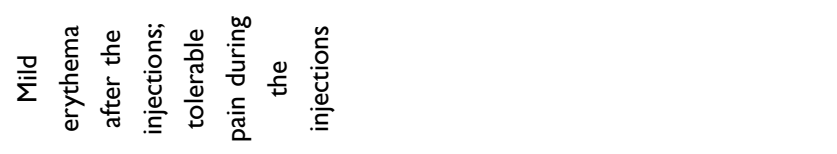 \\
\hline & 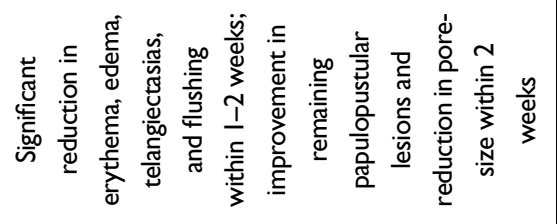 & 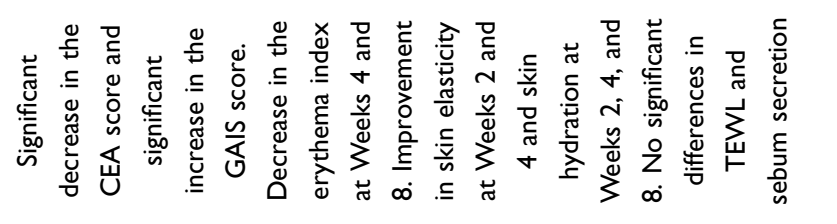 \\
\hline 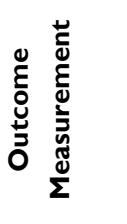 & 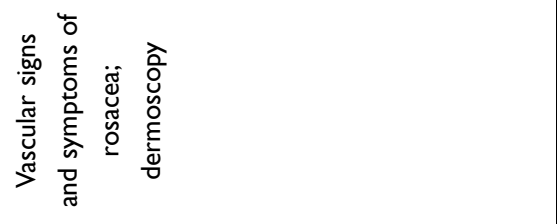 & 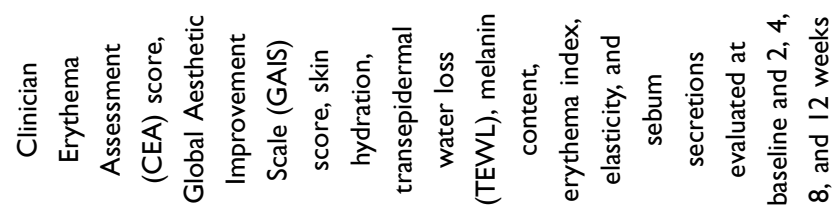 \\
\hline 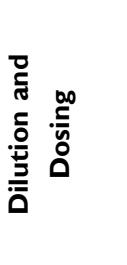 & 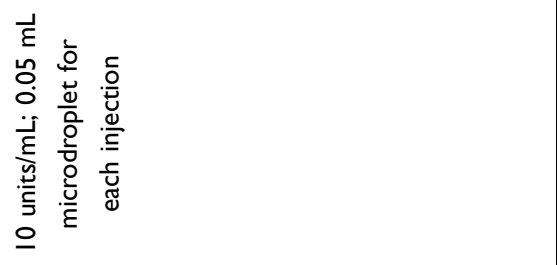 & 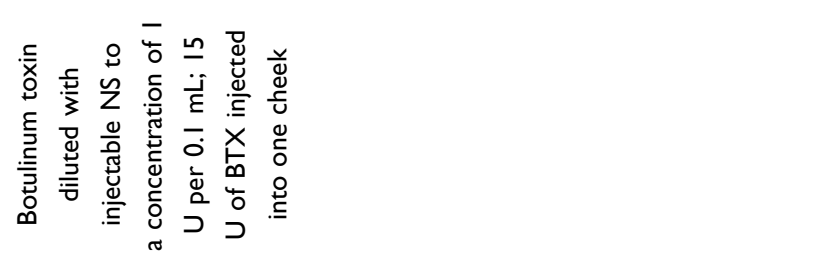 \\
\hline 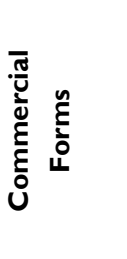 & 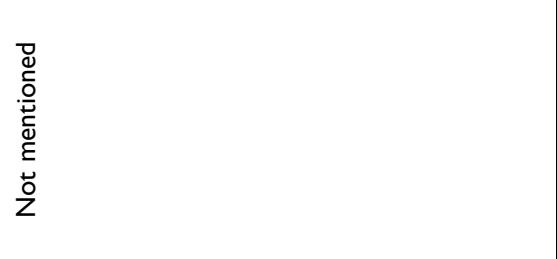 & 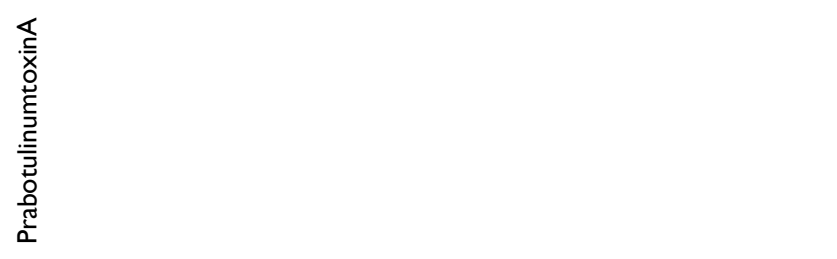 \\
\hline 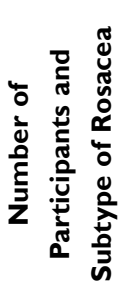 & 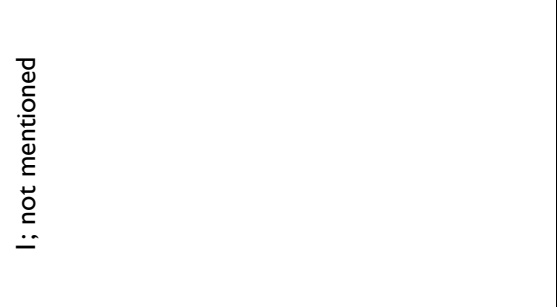 & 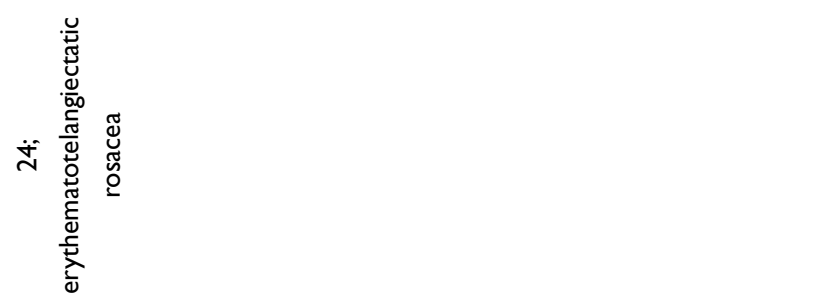 \\
\hline 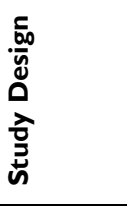 & 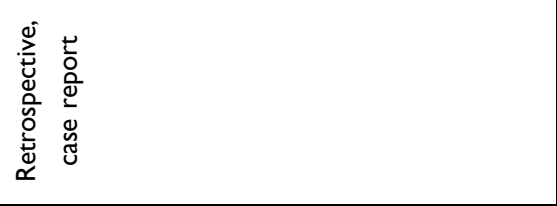 & 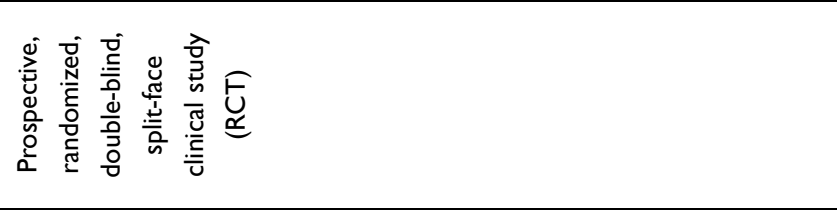 \\
\hline 竞 & 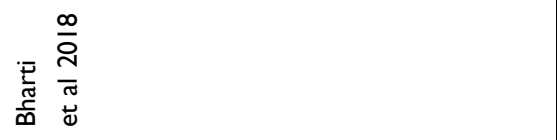 & 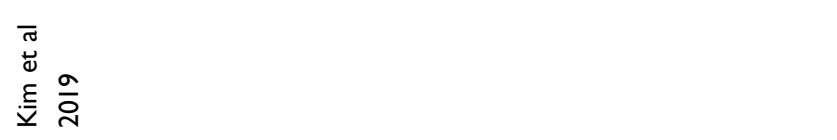 \\
\hline
\end{tabular}




\begin{tabular}{|c|c|}
\hline$\stackrel{\nabla}{\stackrel{\nabla}{\Xi}}$ & $\frac{⿱}{\stackrel{v}{J}}$ \\
\hline 3 & 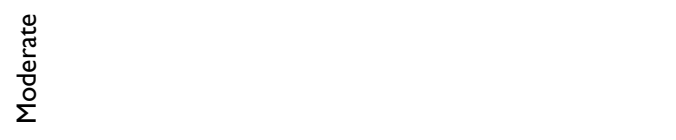 \\
\hline ○ & $\sigma \stackrel{\substack{\underline{E} \\
\stackrel{n}{0}}}{E}$ \\
\hline 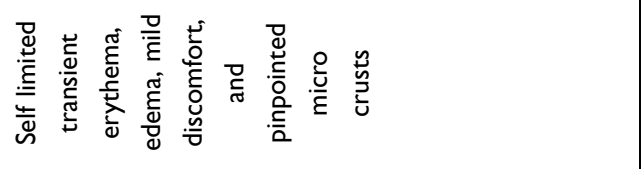 & 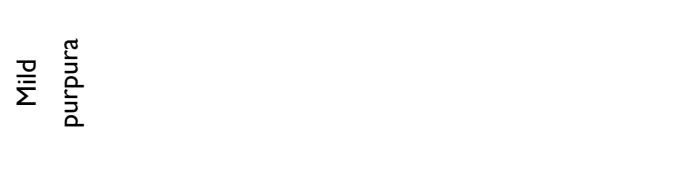 \\
\hline 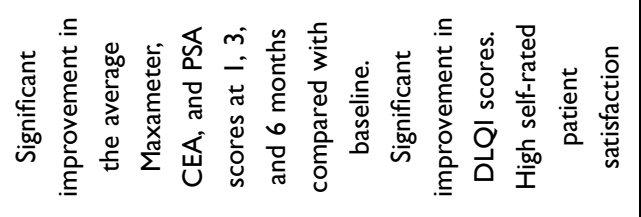 & 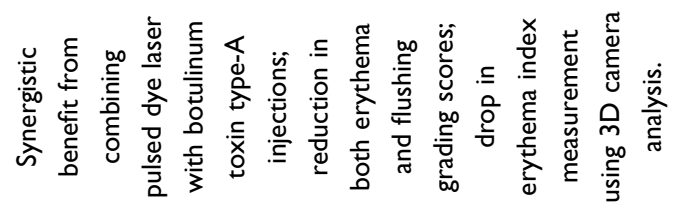 \\
\hline 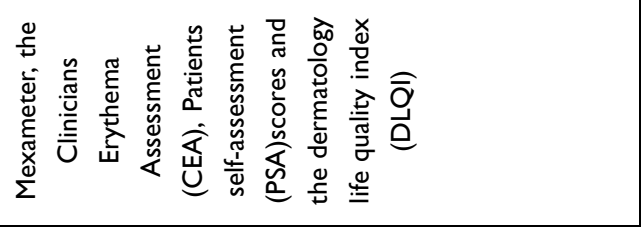 & 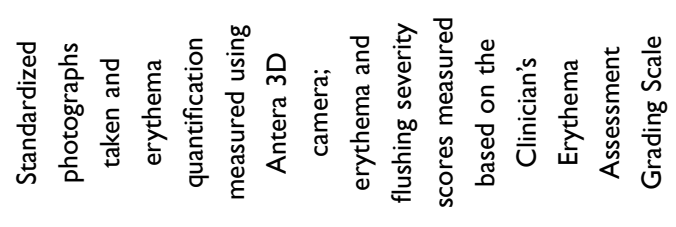 \\
\hline 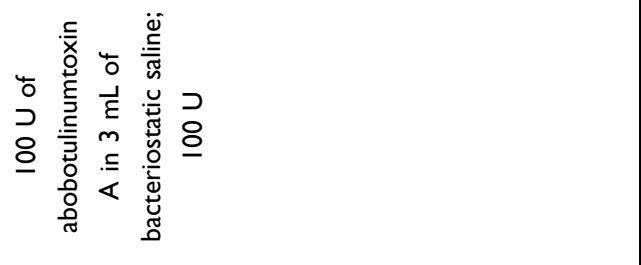 & 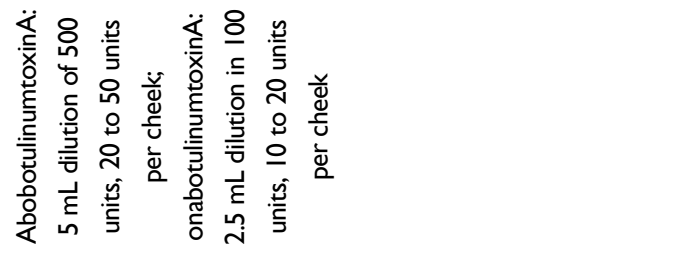 \\
\hline 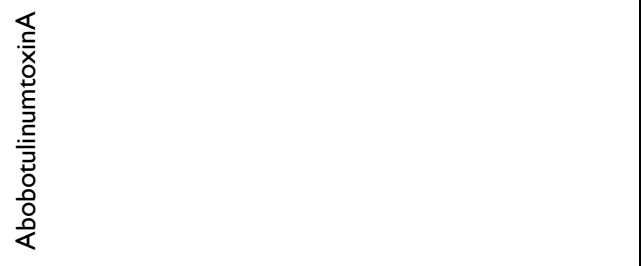 & 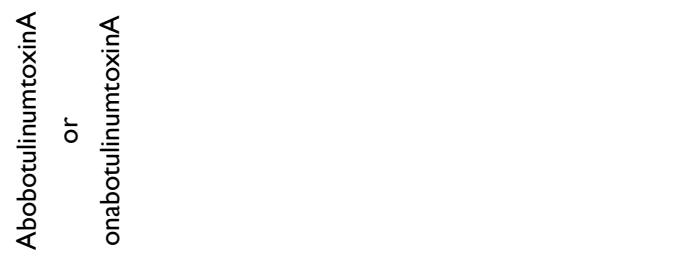 \\
\hline 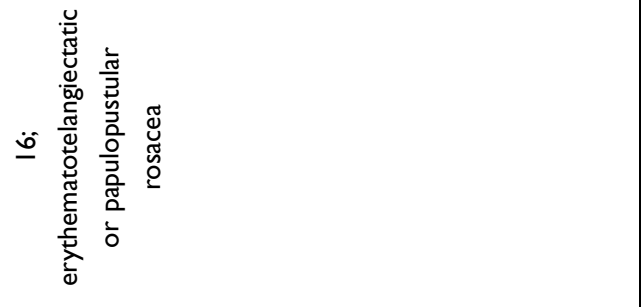 & 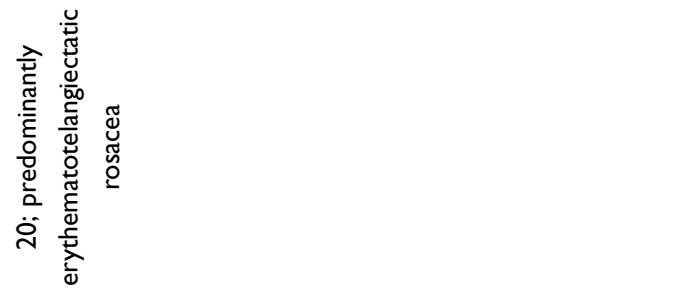 \\
\hline 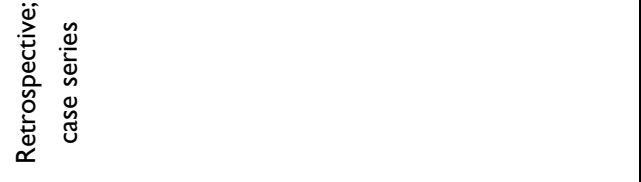 & 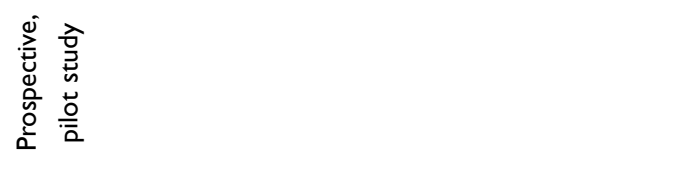 \\
\hline 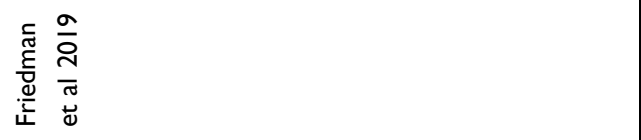 & 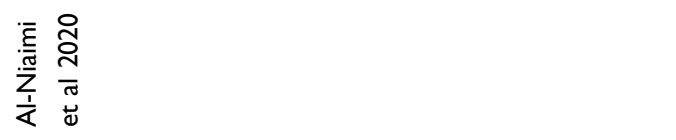 \\
\hline
\end{tabular}


challenging in rosacea treatment. ${ }^{12,40}$ Botulinum toxin, especially type A, has many off-label uses in aesthetic treatments. To date, nine articles described the use of botulinum toxin in treating rosacea, in which Dayan et al from the USA, and Park et al from Korea contributed two, respectively.

Of all the nine studies, only one did not mention the formulation of botulinum toxin. Four different formulations were used in the other eight studies. Different studies applied different outcome measurements, including qualitative and quantitative evaluations from doctors or patients. In the beginning, only signs, symptoms, and personal satisfaction were described in the case report without using the scale method. ${ }^{27}$ Gradually, previously reported scales, such as Rosacea Clinical Scorecard (RCS), ${ }^{30,41}$ Global Aesthetic Improvement Scale (GAIS), ${ }^{33,42}$ were used to assess the severity of rosacea symptoms. Mexameter MX18 ${ }^{31}$, dermoscopy, ${ }^{32}$ and a combination corneometer/mexameter/ reviscometer/sebumeter device ${ }^{33}$ were used to evaluate therapeutic responses. State Self-Esteem Scale (SSES) ${ }^{30,43}$ was used to assess subjects' self-esteem. These ways to measure therapeutic outcomes may be referenced in future studies.

In all the nine studies, overall satisfactory therapeutic effects were achieved, in the perspective of both dermatologists and patients. Side effects mentioned in these articles were mild and self-limited. Of note is that in Bloom et al study, ${ }^{28}$ one withdrew, and nine had inconsistent follow-up visits. They did not follow up possibly due to the dissatisfaction of treatment effects, or potential concern about adverse effects. In Park et al study, ${ }^{31}$ facial muscle paralysis was reported in three patients, and resolved without any special treatment. This was possibly due to treatment volume and injection depth. The longest follow-up in these studies was only 9 months, which might still not enough to evaluate the long-term treatment effects and adverse effects.

Study design can determine the quality of clinical study to a large extent. Randomized controlled trial is superior to others with the least biased results. ${ }^{44}$ In the nine studies, only two studies were randomized controlled trials. A prospective, pilot, double-blind, placebo-controlled study ${ }^{30}$ published in 2017 and a randomized, double-blind, placebo-controlled, splitface pilot study ${ }^{33}$ published in 2019 both assessed the efficacy and safety of botulinum toxin injections in rosacea treatment. Although the other seven studies were all Level 4, with lowgrade clinical evidence, they all showed overall satisfying therapeutic effects. However, none of the included articles had over 25 subjects, with even smaller intervention or control groups. Some were just case reports or case series without control group. ${ }^{29,32}$ The small sample size might also decrease the level of evidence in these studies.

Assessment of bias risk might help to interpret the validity and reliability of the included studies. $77.8 \%$ of studies $(n=7)$ were of low to moderate risk of bias, which presented the effects of botulinum toxin appropriately in treating rosacea. Concerning two potentially high-risk studies, Bharti et al case report did not clearly describe the patient's demographic characteristics, history, and adverse events, possibly because this article type (Therapeutic Pearl) had an upper word limits. ${ }^{32}$ In Kim et al study, the funds and drugs provided by a pharmaceutical company introduced a high risk. ${ }^{33}$ These risk factors must be considered when a researcher is interpreting study findings, which may help to improve study design in the future.

Unfortunately, a meta-analysis could not be conducted, because of small sample size, variation in study types and outcome measurements, lack of control groups, and lack of specific data. Combining outcomes in different types of studies or conducting subgroup analysis is not recommended. ${ }^{45}$ Outcomes in two RCTs cannot be combined to conduct the quantitative analysis, since they were evaluated using different methods, and part of specific data needed was not provided. A unified outcome measurement, from the perspective of both dermatologists and patients, should be established and applied in further studies.

Of all the nine included studies, eight used the intradermal injection of botulinum toxin, and only one used a novel thermomechano-ablative device followed immediately by topical application of botulinum toxin assisted by ultrasound impact system. ${ }^{13}$ Botulinum toxin molecules, unless disrupted, have difficulty in penetrating the stratum corneum because of its characteristics and high molecular weight. ${ }^{13}$ Scientists are trying to provide some conventionally injectable drugs with the ability of transdermal delivery, in the form of gel, cream, ointments, and even ready-to-use pads, using nanotechnology and other technologies. ${ }^{46,47}$ Altering drug or vehicle interaction, using trans-epidermal carriers, modifying or removing stratum corneum (eg, microneedle, laser), and using electric field are all techniques to enhance the ability of transdermal delivery. ${ }^{13}$ The efficacy and safety of topical botulinum toxin were verified in treating primary axillary hyperhidrosis, ${ }^{48}$ hyper-functional wrinkles, ${ }^{49}$ and lateral canthal lines ${ }^{50}$ in several clinical trials. A right-left comparison trial about laser-assisted drug delivery of botulinum toxin on palmar sweat was conducted. ${ }^{51}$ No statistically significant reduction of sweating was observed between the two sides, but pain intensity was higher on the side treated 
with the intradermal injection. The combination of botulinum toxin and laser- or light-based therapies received satisfying therapeutic effects. ${ }^{52}$ In 2020, Al-Niaimi et al used pulsed dye laser followed by intradermal botulinum toxin to treat rosacea-associated erythema and flushing. ${ }^{34}$ A synergistic benefit from the combination therapy was observed: erythema index measured by 3D camera was dropped, and the erythema and flushing grading scores were reduced. Combination therapy in rosacea treatment was also used and recommended in other recent studies, which needs further evaluation and verification. ${ }^{53,54}$

Mechanisms by which botulinum toxin improves rosacea symptoms should be elucidated. ${ }^{28}$ In our searching process, one article on the mechanism of botulinum toxin injections on refractory rosacea was identified. ${ }^{55}$ In this article, researchers found that botulinum toxin could reduce rosacea by inhibiting mast cell degranulation, based on mouse experiments, real-time quantitative RT-PCR, mast cell degranulation assay, and histologic analysis. However, in the nine articles, no mechanisms were clearly verified. Some researchers only reviewed the possible mechanisms in previous literatures, and identified some key substances, such as acetylcholine, ${ }^{27}$ vasoactive intestinal polypeptide, vascular endothelial growth factor, substance $\mathrm{P}$, calcitonin generelated peptide, and transient receptor potential channel vanilloid family member 1 receptors. ${ }^{27,31,33}$

This study has several limitations. First, although four databases were searched, some studies meeting the inclusion criterion may not be included. Second, the included studies had limited sample sizes and heterogeneous designs and outcome measurements. Meta-analysis could not be performed because of the heterogeneity. This limitation makes it hard to attain a generalized conclusion on the use of botulinum toxin in treating rosacea. Thus, we only systematically reviewed this research area, hoping to provide a reference and pave the way for further studies, such as RCTs, mechanisms, or combination therapy.

\section{Conclusions}

This study updated and systematically reviewed the use of botulinum toxin in treating rosacea. The available research showed that the botulinum toxin could have overall satisfying efficacy and safety in the treatment of rosacea, though limited by small sample size, imperfect study design, and short follow-up visits. This systematic review may pave the way for further research, and may be useful in decision-making regarding botulinum toxin in treating rosacea. Larger, randomized, placebo-controlled studies with longer follow-up visits are warranted. The optimal dilution and dosing should be determined in further research, though they largely depend on the severity of symptoms. Therapeutic effects of different formulations and on different subtypes of rosacea should be identified, and proper outcome measurements should also be introduced or applied. Additionally, further research is needed, to illuminate molecular mechanisms of botulinum toxin in treating rosacea, and to evaluate the efficacy and safety of relevant combination therapies including botulinum toxin.

\section{Disclosure}

Hanlin Zhang and Keyun Tang are co-first authors. The authors report no conflicts of interest in this work.

\section{References}

1. Buechner SA. Rosacea: an update. Dermatology. 2005;210 (2):100-108. doi:10.1159/000082564

2. Powell FC. Rosacea. New Engl J Med. 2005;352(8):793-803. doi:10.1056/NEJMcp042829

3. Wang Y, Zhang H, Fang R, Tang K, Sun Q. The top 100 most cited articles in rosacea: a bibliometric analysis. J Eur Acad Dermatol Venereol. 2020.

4. Zhang H, Tang K, Wang Y, Fang R, Sun Q. Rosacea and its comorbidities: should be emphasized but should not be overemphasized. J Cosmet Dermatol. 2020;19(12):3414-3415. doi:10.1111/jocd.13793

5. Maggioni D, Cimicata A, Praticò A, et al. A preliminary clinical evaluation of a topical product for reducing slight rosacea imperfections. Clin Cosmet Investig Dermatol. 2020;13:299-308. doi: $10.2147 /$ CCID.S240784

6. Wilkin J, Dahl M, Detmar M, et al. Standard classification of rosacea: report of the national rosacea society expert committee on the classification and staging of Rosacea. J Am Acad Dermatol. 2002;46 (4):584-587. doi:10.1067/mjd.2002.120625

7. Tan J, Almeida LM, Bewley A, et al. Updating the diagnosis, classification and assessment of rosacea: recommendations from the global ROSacea COnsensus (ROSCO) panel. Br J Dermatol. 2017;176 (2):431-438. doi:10.1111/bjd.15122

8. Gallo RL, Granstein RD, Kang S, et al. Standard classification and pathophysiology of rosacea: the 2017 update by the National Rosacea Society Expert Committee. J Am Acad Dermatol. 2018;78 (1):148-155. doi:10.1016/j.jaad.2017.08.037

9. Pelle MT, Crawford GH, James WD. Rosacea: II. Therapy. $J$ Am Acad Dermatol. 2004;51(4):499-512. doi:10.1016/j.jaad.2004.03.033

10. Abokwidir M, Feldman SR. Rosacea management. Skin Appendage Disorders. 2016;2(1-2):26-34. doi:10.1159/000446215

11. Berardesca E, Bonfigli A, Cribier B, et al. A split-face study assessing the clinical benefit, tolerability and subject satisfaction of a dermocosmetic in subjects with rosacea associated with erythema and sensitive skin. Clin Cosmet Investig Dermatol. 2020;13:751-758. doi:10.2147/CCID.S266879

12. Park JY, Ahn MK, Cho EB, Park EJ, Kim KH. Dual-frequency ultrasound as a new treatment modality for refractory rosacea: a retrospective study. Dermatol Surg. 2018;44(9):1209-1215. doi:10.1097/DSS.0000000000001552

13. Friedman O, Koren A, Niv R, Mehrabi JN, Artzi O. The toxic edge-A novel treatment for refractory erythema and flushing of rosacea. Lasers Surg Med. 2019;51(4):325-331. doi:10.1002/ $1 \mathrm{sm} .23023$ 
14. Wang Y, Zhang H, Zheng Q, Tang K, Fang R, Sun Q. Botulinum toxin as a double-edged sword in alopecia: a systematic review. J Cosmet Dermatol. 2020;19(10):2560-2565. doi:10.1111/jocd.13647

15. Zhang H, Tang K, Wang Y, Fang R, Sun Q. Botulinum toxin in treating Hailey-Hailey disease: a systematic review. $J$ Cosmet Dermatol. 2021. doi:10.1111/jocd.13963

16. Kasyanju Carrero LM, Ma WW, Liu HF, Yin XF, Zhou BR. Botulinum toxin type A for the treatment and prevention of hypertrophic scars and keloids: updated review. $J$ Cosmet Dermatol. 2019;18(1):10-15. doi:10.1111/jocd.12828

17. Alster TS, Harrison IS. Alternative Clinical Indications of Botulinum Toxin. Am J Clin Dermatol. 2020;21(6):855-880. doi:10.1007/ s40257-020-00532-0

18. Satriyasa BK. Botulinum toxin (Botox) A for reducing the appearance of facial wrinkles: a literature review of clinical use and pharmacological aspect. Clin Cosmet Investig Dermatol. 2019;12:223-228. doi:10.2147/CCID.S202919

19. Yi DJ, Hwang S, Son J, Yushmanova I, Anson Spenta K, St Rose S. real-world safety and effectiveness of onabotulinumtoxina treatment of crow's feet lines and glabellar lines: results of a Korean postmarketing surveillance study. Clin Cosmet Investig Dermatol. 2019;12:851-856. doi:10.2147/CCID.S227493

20. Weinkle AP, Doktor V, Emer J. Update on the management of rosacea. Plast Surg Nurs. 2015;35(4):184-202. doi:10.1097/ PSN.0000000000000111

21. Schlessinger J, Gilbert E, Cohen JL, Kaufman J. New uses of AbobotulinumtoxinA in aesthetics. Aesthet Surg J. 2017;37 (suppl_1):S45-s58. doi:10.1093/asj/sjx005

22. Moher D, Liberati A, Tetzlaff J, Altman DG. Preferred reporting items for systematic reviews and meta-analyses: the PRISMA statement. BMJ (Clinical Research Ed). 2009;339:b2535. doi:10.1136/bmj.b2535

23. Higgins JPT, Altman DG, Gøtzsche PC, et al. The Cochrane Collaboration's tool for assessing risk of bias in randomised trials. BMJ. 2011;343(oct18 2):d5928. doi:10.1136/bmj.d5928

24. Slim K, Nini E, Forestier D, Kwiatkowski F, Panis Y, Chipponi J. Methodological index for non-randomized studies (minors): development and validation of a new instrument. ANZ J Surg. 2003;73 (9):712-716. doi:10.1046/j.1445-2197.2003.02748.x

25. Moola S, Munn Z, Tufanaru C, et al. Chapter 7: Systematic Reviews of Etiology and Risk. Joanna Briggs Institute Reviewer's Manual. The Joanna Briggs Institute; 2017.

26. Negoi I, Beuran M, Hostiuc S, Negoi RI, Inoue Y. Surgical anatomy of the superior mesenteric vessels related to colon and pancreatic surgery: a systematic review and meta-analysis. Sci Rep. 2018;8 (1):4184. doi:10.1038/s41598-018-22641-x

27. Dayan SH, Pritzker RN, Arkins JP. A new treatment regimen for rosacea: onabotulinumtoxinA. Journal of Drugs in dermatology: $J D D$. 2012;11(12):e76-79.

28. Bloom BS, Payongayong L, Mourin A, Goldberg DJ. Impact of intradermal abobotulinumtoxinA on facial erythema of rosacea. Dermatol Surg. 2015;41(Suppl 1):S9-16. doi:10.1097/ DSS.0000000000000277

29. Park KY, Hyun MY, Jeong SY, Kim BJ, Kim MN, Hong CK. Botulinum toxin for the treatment of refractory erythema and flushing of rosacea. Dermatology (Basel, Switzerland). 2015;230(4):299-301. doi: $10.1159 / 000368773$

30. Dayan SH, Ashourian N, Cho K, Pilot A. Double-blind, placebo-controlled study to assess the efficacy and safety of IncobotulinumtoxinA injections in the treatment of Rosacea. Journal of Drugs in Dermatology: JDD. 2017;16(6):549-554.

31. Park KY, Kwon HJ, Kim JM, et al. A pilot study to evaluate the efficacy and safety of treatment with botulinum toxin in patients with recalcitrant and persistent erythematotelangiectatic Rosacea. Ann Dermatol. 2018;30(6):688. doi:10.5021/ad.2018.30.6.688
32. Bharti J, Sonthalia S, Jakhar D. Mesotherapy with Botulinum toxin for the treatment of refractory vascular and papulopustular rosacea. J Am Acad Dermatol. 2018. doi:10.1016/j.jaad.2018.05.014

33. Kim MJ, Kim JH, Cheon HI, et al. Assessment of skin physiology change and safety after intradermal injections with botulinum toxin: a randomized, double-blind, placebo-controlled, split-face pilot study in Rosacea patients with facial erythema. Dermatol Surg. 2019;45 (9):1155-1162. doi:10.1097/DSS.0000000000001819

34. Al-Niaimi F, Glagoleva E, Araviiskaia E. Pulsed dye laser followed by intradermal botulinum toxin type- $A$ in the treatment of rosacea-associated erythema and flushing. Dermatol Ther. 2020;33 (6):e13976. doi:10.1111/dth.13976

35. Dayan SH, Ashourian N, Cho K, Pilot A. Double-blind, placebo-controlled study to assess the efficacy and safety of incobotulinumtoxinA injections in the treatment of rosacea. $J$ Drugs Dermatol. 2017;16(6):549-554.

36. Engin B, Özkoca D, Kutlubay Z, Serdaroğlu S. Conventional and novel treatment modalities in Rosacea. Clin Cosmet Investig Dermatol. 2020;13:179-186. doi:10.2147/CCID.S194074

37. Dursun R, Daye M, Durmaz K. Acne and rosacea: what's new for treatment? Dermatol Ther. 2019;32(5):e13020. doi:10.1111/dth.13020

38. Zhang H, Tang K, Wang Y, Fang R, Sun Q. Rosacea treatment: review and update. Dermatol Ther (Heidelb). 2021;11(1):13-24. doi:10.1007/s13555-020-00461-0

39. Juliandri J, Wang X, Liu Z, Zhang J, Xu Y, Yuan C. Global rosacea treatment guidelines and expert consensus points: the differences. J Cosmet Dermatol. 2019;18(4):960-965. doi:10.1111/jocd.12903

40. Gold MH, Lebwohl M, Biesman BS, et al. Daily oxymetazoline cream demonstrates high and sustained efficacy in patients with persistent erythema of rosacea through 52 weeks of treatment. $J \mathrm{Am}$ Acad Dermatol. 2018;79(3):e57-e59. doi:10.1016/j.jaad.2018.05.037

41. Wilkin J, Dahl M, Detmar M, et al. Standard grading system for rosacea: report of the National Rosacea Society Expert Committee on the classification and staging of rosacea. J Am Acad Dermatol. 2004;50(6):907-912. doi:10.1016/j.jaad.2004.01.048

42. Roh NK, Kim MJ, Lee YW, Choe YB, Ahn KJ. A split-face study of the effects of a stabilized hyaluronic acid-based gel of nonanimal origin for facial skin rejuvenation using a stamp-type multineedle injector: a randomized clinical trial. Plast Reconstr Surg. 2016;137 (3):809-816. doi:10.1097/01.prs.0000480686.68275.60

43. Heatherton TF, Development PJ. Validation of a Scale for Measuring State Self-Esteem. J Pers Soc Psychol. 1991;60(6):895-910. doi:10.1037/0022-3514.60.6.895

44. Fukui T, Rahman M, Sekimoto M, et al. Study design, statistical method, and level of evidence in Japanese and American clinical journals. J Epidemiol. 2002;12(3):266-270. doi:10.2188/jea.12.266

45. Higgins JPT, Thomas J, Chandler J, et al. Cochrane handbook for systematic reviews of interventions version 6.0 (updated July 2019). Cochrane. 2019.

46. Saffarian P, Fooladi AAI. Topical botulinum toxin: a non-invasive way for treatment of muscle disorders. Curr Drug Deliv. 2018;15 (10):1375-1380. doi:10.2174/1567201815666180730140704

47. Collins A, Nasir A. Topical botulinum toxin. J Clin Aesthet Dermatol. 2010;3(3):35-39.

48. Lueangarun S, Sermsilp C, Tempark T. Topical Botulinum Toxin Type A liposomal cream for primary axillary hyperhidrosis: a double-blind, randomized, split-site, vehicle-controlled study. Dermatol Surg. 2018;44(8):1094-1101. doi:10.1097/DSS.0000000000001532

49. Chajchir I, Modi P, Chajchir A. Novel topical BoNTA (CosmeTox, toxin type A) cream used to treat hyperfunctional wrinkles of the face, mouth, and neck. Aesthetic Plast Surg. 2008;32(5):715-722; discussion 723. doi:10.1007/s00266-008-9151-9

50. Glogau R, Blitzer A, Brandt F, Kane M, Monheit GD, Waugh JM. Results of a randomized, double-blind, placebo-controlled study to evaluate the efficacy and safety of a botulinum toxin type A topical gel for the treatment of moderate-to-severe lateral canthal lines. Journal of Drugs in Dermatology: JDD. 2012;11(1):38-45. 
51. Issa MC, Torreao PS, Boechat M, Luiz R. Early investigations in drug delivery of onabotulinum toxin A using combined fractional ablative laser with impact ultrasound vs. injections of onabotulinum toxin A for palmar hyperhidrosis: a right-left comparison trial. $\mathrm{Br}$ J Dermatol. 2018;179(5):1168-1169. doi:10.1111/bjd.16781

52. Zhu J, Ji X, Li M, et al. The efficacy and safety of fractional $\mathrm{CO}_{2}$ laser combined with topical Type A botulinum toxin for facial rejuvenation: a randomized controlled split-face study. Biomed Res Int. 2016;2016:3853754. doi:10.1155/2016/3853754

53. Schilling LM, Halvorson CR, Weiss RA, Weiss MA, Beasley KL. Safety of combination laser or intense pulsed light therapies and doxycycline for the treatment of Rosacea. Dermatol Surg. 2019;45 (11):1401-1405. doi:10.1097/DSS.0000000000002009
54. Ebneyamin E, Mansouri P, Rajabi M, Qomi M, Asgharian R, Azizian Z. The efficacy and safety of permethrin $2.5 \%$ with tea tree oil gel on rosacea treatment: a double-blind, controlled clinical trial. J Cosmet Dermatol. 2019;19(6):1426-1431. doi:10.1111/jocd.13177

55. Choi JE, Werbel T, Wang Z, Wu CC, Yaksh TL, Di Nardo A. Botulinum toxin blocks mast cells and prevents rosacea like inflammation. J Dermatol Sci. 2019;93(1):58-64. doi:10.1016/j. jdermsci.2018.12.004

\section{Publish your work in this journal}

Clinical, Cosmetic and Investigational Dermatology is an international, peer-reviewed, open access, online journal that focuses on the latest clinical and experimental research in all aspects of skin disease and cosmetic interventions. This journal is indexed on CAS.
The manuscript management system is completely online and includes a very quick and fair peer-review system, which is all easy to use. Visit http://www.dovepress.com/testimonials.php to read real quotes from published authors. 